\section{Schweizerische Gesellschaft für Innere Medizin}

\section{Société Suisse de Médecine Interne}

Vorstand

Präsident:

Prof. Dr. med. F. Follath, Zürich*

President elect:

Dr. med. W. Bauer, Küsnacht ${ }^{+}$

Vizepräsident/Vorsitz API:

Dr. med. J. Fritschi, Pfeffingen ${ }^{+}$

Tagungspräsident 2001:

Prof. Dr. A. Pécoud, Lausanne*

Aktuarin:

PD Dr. med. V. Briner, Luzern*

Quästorin:

Dr. med. S. Keberle, Basel ${ }^{+}$

Mitglieder:

Dr. med. B. Buchs, Genève+*

PD Dr. med. R. Malinverni, Neuchâtel*;

Prof. Dr. med. P. Nicod, Lausanne*;

Dr. med. B. Roessler, Niederbipp ${ }^{+}$;

Dr. med. R. Schibli, Lachen ${ }^{+}$;

Prof. Dr. med. J. Schifferli, Basel*;

Dr. med. M. Schneller, Sierre ${ }^{+}$;

Dr. med. E. Simon, Lausanne ${ }^{+}$;

Prof. Dr. med. Max Stäubli, Zollikerberg*;

Frau Dr. med. S. Stöhr, Basel*;

Prof. Dr. med. F. Waldvogel, Genève*

Sekretariat:

SGIM, Rotbergerstrasse 34, 4011 Basel,

Tel. 06122593 30, Fax 06122593 31,

E-mail: info@sgim.ch

* Mitglieder des Ausschusses

der Spitalinternisten (ASI)

+ Mitglieder des Ausschusses

der praktizierenden Internisten (API)

\section{Schweizerische Gesellschaft für Radiologie}

\section{Société Suisse de Radiologie}

Vorstand 2000/2002

Präsident:

PD Dr. C. Becker, Genf

Designierter Präsident:

PD Dr. H. M. Hoogewoud, Freiburg

Generalsekretär:

Prof. Dr. P. Probst, Solothurn

Kassier:

PD Dr. H. M. Hoogewoud, Freiburg

Schriftführer:

Dr. S. Paul, Biel / Dr. D. Toia, Liestal

Beisitzer:

Dr. F. Bossard (Past President), Thun;

Dr. M. Lütolf, Chur;

Dr. P. Chevalley, Freiburg

Sekretariat SGR-SSR:

Frau A. Molo, Tel./Fax 03130122 55,

E-mail: sgr-ssr@thenet.ch

\section{Schweizerische Gesellschaft für Chirurgie \\ Société Suisse de Chirurgie}

Vorstand/Comité 2000-2002

Präsident:

Prof. Dr. Jean Claude Givel, Lausanne

Vizepräsident \&t Ressortleiter

für Wissenschaft:

Prof. Dr. Markus Wolfgang Büchler, Bern

Altpräsident:

Prof. Dr. Thomas Rüedi, Chur

Ressortleiter Berufsausübung:

Dr. med. Gabriel Ayer, Wil

Dr. med. Luca Rondi, Lugano

Ressortleiter Berufsbildung:

PD Dr. med. Rolf Schlumpf, Aarau

Ressortleiter Qualitätsmanagement: PD Dr. med. Walter Schweizer,

Schaffhausen

Beisitzer:

Prof. Dr. Pietro Regazzoni, Basel

Generalsekretär (ex officio):

Dr. med. Jean Biaggi, Muri

Gesellschaftssekretariat:

SGC Sekretariat, Netzibodenstrasse 34, Postfach 1527, 4133 Pratteln 1,

Tel. 06181596 60, Fax 06181147 75,

E-mail: info@sgc-ssc.ch,

Homepage: www.sgc-ssc.ch 


\section{Information betreffend Fehldruck auf der Blisterfolie}

Creon forte, 50 und 100 Kapseln, IKS-Nr. 38'219; betroffene Chargen: 12361 (50erPackungen), 12360 und 13193 (100erPackungen)

Bei den oben genannten Chargen sind die Blister falsch bedruckt: anstatt richtig "creon forte, 300mg Pankreatin" steht irrtümlicherweise "creon forte $150 \mathrm{mg}$ Pankreatin". Die Ware ist dagegen in Ordnung, und die Angaben auf den Faltschachteln und den Packungsbeilagen sind korrekt.

Wir rufen deshalb alle Packungen dieser drei Chargen auf Stufe Grosshandel zurück und tauschen sie - solange noch keine neuen Chargen mit korrekt beschrifteten Blistern geliefert werden können - gegen Packungen derselben Chargen aus, die mit einem Kleber mit entsprechendem Hinweis versehen sind.

Allfällige bei Ihnen noch vorhandene Packungen brauchen nicht zurückgeschickt zu werden. Wir danken für Ihr Verständnis.

Solvay Pharma AG,

Untermattweg 8, 3027 Bern

\section{Information concernant erreur d'imprimerie sur la feuille alu blister}

Creon forte, 50 et 100 capsules, no. OICM 38'219; lots concernés: 12361 (emballages de 50), 12360 et 13193 (emballages de 100)

La feuille alu blister des lots susmentionnés contient une erreur d'imprimerie: au lieu de correctement "creon forte, $300 \mathrm{mg}$ Pankreatin" il est indiqué incorrectement "creon forte, $150 \mathrm{mg}$ Pankreatin". La marchandise néanmoins est irréprochable, et les données sur les étuis et les notices d'emballage sont correctes.

Pour cette raison, nous rappelons tous les emballages des ces trois lots au niveau grossistes et les remplacerons - tant que de nouveaux lots correctement imprimé ne seront pas disponibles - contre des emballages des même lots munis d'une étiquette comportant une information adéquate.

De tels emballages, se trouvant éventuellement encore en votre possession, ne doivent pas être renvoyés. Nous vous remercions de votre compréhension.

Solvay Pharma SA,

Untermattweg 8, 3027 Berne

\section{Chargenrückruf Diclosifar 50 mg, Filmtabletten IKS-Nr. 52'999}

Charge Nr. 1057

Im Rahmen einer periodischen Kontrolle der IKS wurde festgestellt, dass bei der obengenannten Charge die Wirkstoffauflösung (Dissolution) nicht gewährleistet ist.

Wir bitten Sie deshalb, uns alle DiclosifarPackungen zu 50 mg x 20 und x 100 mit der Chargen-Nr. 1057 zurückzusenden, sofern diese Ware bei Ihnen noch vorrätig ist. Dieser Rückruf betrifft lediglich die oben erwähnte Charge unseres Produktes Diclosifar $50 \mathrm{mg}$; alle anderen Packungen von Diclosifar $50 \mathrm{mg}$ (mit der Chargen-Nr. 18058), welche sich im Markt befinden, sind konform und können ohne weiteres verwendet werden.

Selbstverständlich werden Ihnen alle an uns zurückgesandten Packungen ersetzt. Die Speditionskosten gehen zu unseren Lasten. Wir danken für Ihr Verständnis.

\section{SIPHAR SA}

6814 Lamone-Cadempino

\section{Schweineinsulin geht - Schweineinsulin kommt}

Die IKS hat drei hochgereinigte tierische Insuline der Reihe Hypurin ${ }^{\circledR}$ porcine der Firma CP Pharma (Schweiz) AG, der Tochtergesellschaft eines englischen, seit 25 Jahren auf tierisches Insulin spezialiserten Insulinherstellers, registriert. Mit der Kassenzulässigkeit werden die Insuline Hypurin ${ }^{\circledR}$ Neutral, NPH ab 1. Juli 2000 sowie Mix ab August 2000 in den Apotheken erhältlich sein. CP Pharma deckt damit die Palette für das Basis-Bolus-System für Typ-1-Diabetes sowie für Typ-2-Diabetes mit ein oder zwei Tagesdosen ab. Die drei Insuline sind für die Pen-Applikation erhältlich und ermöglichen so Bequemlichkeit in Verbindung mit dem Gefühl von Sicherheit und Wohlbefinden. Die Mitteilung der Firma Novo Nordisk vom 9. Februar 2000, ihre bisherige Produktion von Schweineinsulin werde in den USA sofort und weltweit bis zum Jahr 2005 eingestellt, hätte noch vor wenigen Wochen das Aus für tierische Insuline in der Schweiz bedeutet: Bis jetzt war Novo Nordisk der einzige Anbieter für Schweineinsulin in der Schweiz. Noch vor kurzem versicherte sie, tierische Insuline «bis weit ins nächste Jahrtausend und solange ein medizinisches Bedürfnis besteht zur Verfügung zu stellen». Die weltweiten Rückzugstendenzen der grossen Insulinhersteller aus dem tierischen Insulinmarkt sind jedoch nicht zu übersehen. Dafür sind wirtschaftliche und nicht medizinische Gründe verantwortlich.

In den Produkteinformationen zu neuen synthetischen Insulinanaloga wird unter Vorsichtsmassnahmen nachträglich ein erhöhtes Risiko unter Humaninsulin für Hypoglykämie und u.a. für die Verschlechterung von diabetischer Retinopathie aufgeführt. Um so wichtiger ist es, dass mit den neu zugelassenen Schweineinsulinen der CP Pharma auch die freie Wahl von natürlichen Insulinen mit Pen ermöglicht wird. Nach 10 Jahren Lobbying unserer Patientenorganisation ist die Versorgung mit tierischen Insulinen in der Schweiz vorerst gesichert.

Prof. Arthur Teuscher Forum Insulin Schweiz (FIS) 